Best of Pflege 
Mit „Best of Pflege“ zeichnet Springer die besten Masterarbeiten und Dissertationen aus dem Bereich Pflege aus. Inhalte aus den etablierten Bereichen der Pflegewissenschaft, Pflegepädagogik, Pflegemanagement oder aus neuen Studienfeldern wie Health Care oder Ambient Assisted Living finden hier eine geeignete Plattform. Die mit Bestnote ausgezeichneten Arbeiten wurden durch Gutachter empfohlen und behandeln aktuelle Themen rund um den Bereich Pflege.

Die Reihe wendet sich an Praktiker und Wissenschaftler gleichermaßen und soll insbesondere auch Nachwuchswissenschaftlern Orientierung geben. 
Markus Hieber

\section{Das transformationale \\ Führungsstilmodell}

Historische Entwicklung und heutige Anwendbarkeit

说 Springer 


\author{
Markus Hieber \\ Berlin, Deutschland
}

Diplomarbeit, Hamburger Fernhochschule, Hamburg 2015

Best of Pflege

ISBN 978-3-658-11469-5

ISBN 978-3-658-11470-1 (eBook)

DOI 10.1007/978-3-658-11470-1

Die Deutsche Nationalbibliothek verzeichnet diese Publikation in der Deutschen Nationalbibliografie; detaillierte bibliografische Daten sind im Internet über http://dnb.d-nb.de abrufbar.

Springer

(C) Springer Fachmedien Wiesbaden 2016

Das Werk einschließlich aller seiner Teile ist urheberrechtlich geschützt. Jede Verwertung, die nicht ausdrücklich vom Urheberrechtsgesetz zugelassen ist, bedarf der vorherigen Zustimmung des Verlags. Das gilt insbesondere für Vervielfältigungen, Bearbeitungen, Übersetzungen, Mikroverfilmungen und die Einspeicherung und Verarbeitung in elektronischen Systemen.

Die Wiedergabe von Gebrauchsnamen, Handelsnamen, Warenbezeichnungen usw. in diesem Werk berechtigt auch ohne besondere Kennzeichnung nicht zu der Annahme, dass solche Namen im Sinne der Warenzeichen- und Markenschutz-Gesetzgebung als frei zu betrachten wären und daher von jedermann benutzt werden dürften.

Der Verlag, die Autoren und die Herausgeber gehen davon aus, dass die Angaben und Informationen in diesem Werk zum Zeitpunkt der Veröffentlichung vollständig und korrekt sind. Weder der Verlag noch die Autoren oder die Herausgeber übernehmen, ausdrücklich oder implizit, Gewähr für den Inhalt des Werkes, etwaige Fehler oder Äußerungen.

Gedruckt auf säurefreiem und chlorfrei gebleichtem Papier

Springer Fachmedien Wiesbaden ist Teil der Fachverlagsgruppe Springer Science+Business Media (www.springer.com) 


\section{Geleitwort}

Die Hamburger Fernhochschule (HFH) bietet berufsbezogene Studiengänge in den Bereichen Gesundheit \& Pflege, Wirtschaft \& Recht sowie Technik an. Ein Fernstudium bedeutet, dass die Studierenden sich vor allem Wissen aus den fachlich hochwertigen Studienheften und ergänzender Literatur aneignen, aber auch in Präsenzveranstaltungen an über 50 Studienzentren in Deutschland, Österreich und der Schweiz ihre Kenntnisse vertiefen können. Der Bologna-Prozess zur Vereinheitlichung der Hochschulabschlüsse in Europa gestattete den Hochschulen und Universitäten Übergangsfristen; die HFH bot daher den Diplomstudiengang Pflegemanagement wegen seiner Beliebtheit noch lange an, während andere Hochschulen und Universitäten schon breitflächig auf Bachelor- und Masterstudiengänge umgestiegen waren. Und so startete die HFH den Diplomstudiengang Pflegemanagement letztmalig im Januar 2011. Bis 2016 ist es möglich, die Prüfungen in diesem Studiengang abzulegen. Inzwischen ist an der HFH der Diplomstudiengang Pflegemanagement durch die konsekutiven Studiengänge Pflegemanagement (B.A.) und Management von Organisationen und Personal im Gesundheitswesen, Master of Arts (M.A.) ersetzt worden.

Ich selbst bin als Diplom-Pädagoge seit Jahrzehnten in der Erwachsenenbildung aktiv und als Dozent bei Präsenzveranstaltungen der Hamburger Fernhochschule tätig; zudem biete ich Workshops und Seminare zu diversen Themen an. Die vorliegende, von mir als Erstgutachter betreute Diplomarbeit wendet sich dem Thema Führung zu. In meinen Lehrveranstaltungen und Workshops wurde mir bewusst, wie schwierig die Führungsaufgabe in der Pflege ist, da die Führenden tagtäglich mit neuen Herausforderungen konfrontiert werden. Hier ergibt sich Lernbedarf nicht nur für die neuen Führungskräfte, sondern auch für Personen, die schon viele Erfahrungen in der Führung sammeln konnten.

Die vorliegende Theorie- bzw. Literaturarbeit unterzieht das in den letzten Jahren immer populärer werdende transformationale bzw. charismatische Führungsstilmodell einer kritischen Prüfung. Das transformationale Führungsstilmodell wird vom Autor historisch hergeleitet, differenziert dargestellt und dann ausgiebig diskutiert. Im sechsten Kapitel erfolgt eine Prüfung, inwieweit der transformationale Führungsstil in Pflegeeinrichtungen Anwendung finden kann.

Wie ich auch schon im Gutachten zur Diplomarbeit hervorhob, hat der Autor eine bemerkenswert gute Diplomarbeit vorgelegt. Die Arbeit ist gut strukturiert und mit 
Tiefgang erarbeitet; die zum Teil sehr anspruchsvollen Quellen wurden sorgfältig ausgewertet. Die wissenschaftlichen Kriterien und inhaltlichen Anforderungen an eine Diplomarbeit werden gut erfüllt.

Ph.Dr. Frank Marks, Erstgutachter 


\section{Inhaltsverzeichnis}

Geleitwort

Tabellenverzeichnis

Abbildungsverzeichnis

Abkürzungsverzeichnis

1 Fragen, Ziele, Methode

1.1 Entwicklung der Fragestellung und Beweisziele

1.2 Zur Methode der Literaturstudie

1.3 Literaturrecherche

2 Grundlagen: Klärung wichtiger Begriffe und Verortung des transformationalen Führungsstil-Modells in einem größeren Kontext

2.1 Definition der Grundbegriffe

2.2 Transformationale und charismatische Führung

2.3 Einteilung der Führungsstiltheorien nach Liebel und Einordnung des transformationalen Führungsstilmodells

3 Herkunft des transformationalen Führungsstilmodells

3.1 Charisma im Urchristentum nach Sohm

3.2 Charismatische Führung nach Weber

3.2.1 Definition des Charisma gemäß Weber

3.2.2 „Wesen“ des Charisma

3.2.3 Charisma als revolutionäre Macht 
3.2.4 Veralltäglichung des Charisma $\quad 15$

3.2.5 Einschätzung von Webers charismatischer Herrschaftstheorie 18

3.3 Transaktionale Führung und ,transforming leadership“ in der Politik nach Burns

3.4 Wiederentdeckung des Charisma-Konzepts

4 Kernaussagen des transformationalen Führungsstilmodells nach Bass

4.1 „The Full Range Of Leadership“-

Die Bandbreite möglicher Führungsstile

4.2 Die 4 I's - Techniken der transformationalen Führung 22

4.2.1 Idealized Influence/Charisma 23

4.2.2 Inspirational Motivation/Inspirierendes Führungsverhalten 24

4.2.3 Individualized Consideration/Individuelle Behandlung 25

4.2.4 Intellectual Stimulation/Geistige Anregung 27

5 Tauglichkeit des transformationalen Führungsstilmodells im Allgemeinen 30

5.1 Argumente für das transformationale Führungsstilmodell

5.1.1 Besondere Eignung der transformationalen Führung in Umbruchzeiten und bei Organisationswandel

5.1.2 Entrationalisierung - Berücksichtigung der emotionalen Bedürfnisse der Mitarbeiter/innen

5.1.3 Steigerung der Leistungen der Mitarbeiter/innen

5.1.4 Empirische Belege für die Effektivität der transformationalen Führung

5.1.5 Charismatische Führung als Chance für Frauen

5.2 Argumente gegen das transformationale Führungsstilmodell 37

5.2.1 Kritik an den empirischen Belegen 39

5.2.2 Offene Fragen 43

5.2.3Kohärenzprüfung des transformationalen Führungsstilmodells $\quad 44$ 
5.2.4 Implizite, problematische Annahmen 46

5.2.5 Problematische Begriffe 48

5.2.6 Ethische Problematik 54

5.2.7 Legendenbildung oder Narzissmus? 58

5.2.8 Weitreichender Eingriff in die Persönlichkeit der Mitarbeiter/innen 61

5.2.9 Romance of Leadership - Überschätzung der Einflussmöglichkeiten $\begin{array}{ll}\text { der Führungspersonen } & 64\end{array}$

5.2.10 Potential für die Besetzung von Führungspositionen mit

$\begin{array}{ll}\text { Charismatikern } & 66\end{array}$

$\begin{array}{ll}\text { 5.2.11 Nachfolgeproblematik } & 68\end{array}$

5.2.12 Dysfunktionalitäten des transformationalen Führungsstilmodells 69

5.2.13 Transformationale Führung in Hinblick

gesellschaftlicher Tendenzen $\quad 69$

5.2.14 Benachteiligung der Frauen in der Literatur zur transformationalen

$\begin{array}{ll}\text { Führung } & 71\end{array}$

5.3 Diskussion 72

5.3.1 Widersprüche in der Kritik 73

5.3.2 Leicht widerlegbare Kritik 74

$\begin{array}{lll}\text { 5.3.3 Gelungene Kritik } & 75\end{array}$

6 Anwendbarkeit der transformationalen Führung in Pflegeeinrichtungen 76

6.1 Kilians Studie zur transformationalen Führung in der Pflege 76

6.2 Situation in den Pflegeeinrichtungen im Allgemeinen 78

6.3 Situation der PDLs im Speziellen 79

6.4 Braucht eine PDL Charisma? 83

7 No More Heroes, No More Shakespearoes - Schlussfolgerungen aus der $\begin{array}{ll}\text { Diskussion und Fazit } & 85\end{array}$

7.1 Kernproblem: Säkularisation eines religiösen Begriffs 85 
7.2 Rettungsversuche

7.3 Abschließende Auswertung

7.4 Die neuen Realitäten: Ausblick und Alternativen

8 Literaturverzeichnis 


\section{Tabellenverzeichnis}

Tab. 1: Arten der Nachfolgeregelung (nach Weber 2013: 498 ff.)

Tab. 2: Kritiker/innen und ihre Themen

Eigene Darstellung.

Tab. 3: Dimensionen zur Messung der transformationalen Führung und Dimensionen zur Messung der Effizienzkriterien

(vgl. Tartler et al. 2004: 260).

Tab. 4: Impressionskontinuum der Führung (vgl. Steyrer 2011: 83). .54

\section{Abbildungsverzeichnis}

Abb. 1: Profile für 2 Aussagen zum MLQ

Quelle: Tartler et al. 2004: 262. .38 


\section{Abkürzungsverzeichnis}

Abkürzung

4 I's

Abb.

AG

aktual.

Aufl.

AZ

BZÖ

bearb.

bzw.

CEO

d. h.

DBfK

Dip

Dipl.-Päd.

Dr.

Effec

Enron

Epis.

ePub

erw.

et al.

etc.

e. V.

ExEff

f.

ff.

FPÖ

Hrsg.

http

II
Bedeutung

die vier Techniken der transformationalen Führung

Abbildung

Aktiengesellschaft

aktualisierte

Auflage

Arbeitszufriedenheit

Bündnis Zukunft Österreich

bearbeitete

beziehungsweise

Chief Executive Officer $=$ geschäftsführendes Vorstandsmitglied

das heißt

Deutscher Berufsverband für Pflegeberufe

Diplomarbeit

Diplom-Pädagoge

Doktor

Effektivness

Abkürzung für „EnterOn“ (ehemaliger Energiekonzern in den USA)

Episode

electronic publication

erweiterte

et alii (Maskulinum), et aliae (Femininum) oder et alia

(Neutrum) $=$ und andere

et cetera $=$ und so weiter

eingetragener Verein

Extra Effort

folgende Seite

folgende Seiten

Freiheitliche Partei Österreichs

Herausgeber

Hypertext Transfer Protocol

Idealized Influence $=$ idealisierte Einflussnahme 
Abkürzung

IC

IIa

$\mathrm{IIb}$

IM

insb.

IS

Jr.

Kap.

KFZ

M. A.

MLQ

n. Chr.

Nachdr.

N. N.

Nr.

NS-Staat

NSDAP

o. S.

passim

PDL

Ph. D.

PM

Prof.

RBS

Sat

sic!

S.

Tab.

u. a.

überarb.
Bedeutung

Individualized Consideration $=$ Individuelle Wertschätzung

Idealized Influence Attributed $=$ zugeschriebene idealisierte Einflussnahme (= Charisma)

Idealized Influence Behavior = idealisierte Einflussnahme im Verhalten (=Visionen etc.)

Inspirational Motivation = Inspirierende Motivierung insbesondere

Intellectual Stimulation = Intellektuelle Anregung Junior

Kapitel

Kraftfahrzeug

Magister Artium

Multifactor Leadership Questionnaire

nach Christus

Nachdruck

Nomen nominandum $=$ lateinisch für „,noch zu nennender Name“"

Nummer

nationalsozialistischer Staat

Nationalsozialistische Deutsche Arbeiterpartei

ohne Seitenzahl

lateinisch für ,überall“

Pflegedienstleitung

englisch „Doctor of Philosophy“, neulateinisch „philosophiae doctor"

Pflegemanagement

Professor

The Royal Bank Of Scotland

Satisfaction

„wirklich so“"

Seiten

Tabelle

unter anderem

überarbeitete 


$\begin{array}{ll}\begin{array}{ll}\text { Abkürzung } \\ \text { unveränd. }\end{array} & \begin{array}{l}\text { Bedeutung } \\ \text { unveränderter } \\ \text { u.v.m. }\end{array} \\ \text { UFA } & \text { und vieles mehr } \\ \text { UFO } & \text { Universum Film AG } \\ & \text { Unidentified Flying Object = unidentifiziertes fliegendes } \\ \text { Objekt } \\ \text { US } & \text { United States } \\ \text { USA } & \text { United States of America = Vereinigte Staaten von Ame- } \\ & \text { rika } \\ \text { v. a. } & \text { vor allem } \\ \text { vgl. } & \text { vergleiche } \\ \text { vollst. } & \text { vollständig } \\ \text { ZAG SL/WBL } & \text { Zentrale Arbeitsgruppe Stations- und Wohnbereichslei- } \\ & \text { tungen } \\ \text { z. B. } & \text { zum Beispiel } \\ \text { zit. } & \text { zitiert }\end{array}$

